\title{
Aerodynamic Modeling of Transonic Aircraft Using Vortex Lattice Coupled with Transonic Small Disturbance for Conceptual Design
}

\author{
Daniel Chaparro * \\ MORI Associates, Moffett Field, CA 94035 \\ Gustavo E. C. Fujiwara ${ }^{\dagger}$ \\ University of Washington, Seattle, WA 98195 \\ Eric Ting ${ }^{\ddagger}$ \\ Stinger Ghaffarian Technologies Inc. Moffett Field, CA 94035 \\ Nhan Nguyen ${ }^{\S}$ \\ NASA Ames Research Center, Moffett Field, CA 94035
}

\begin{abstract}
The need to rapidly scan large design spaces during conceptual design calls for computationally inexpensive tools such as the vortex lattice method (VLM). Although some VLM tools, such as Vorview have been extended to model fully-supersonic flow, VLM solutions are typically limited to inviscid, subcritical flow regimes. Many transport aircraft operate at transonic speeds, which limits the applicability of VLM for such applications. This paper presents a novel approach to correct three-dimensional VLM through coupling of two-dimensional transonic small disturbance (TSD) solutions along the span of an aircraft wing in order to accurately predict transonic aerodynamic loading and wave drag for transport aircraft. The approach is extended to predict flow separation and capture the attenuation of aerodynamic forces due to boundary layer viscosity by coupling the TSD solver with an integral boundary layer (IBL) model. The modeling framework is applied to the NASA General Transport Model (GTM) integrated with a novel control surface known as the Variable Camber Continuous Trailing Edge Flap (VCCTEF).
\end{abstract}

\section{Introduction}

The commercial transport industry is trending towards incorporating composite materials and other lightweight materials with the goal of achieving more energy-efficient aircraft. Weight reduction leads to a lower lift requirements and subsequently reductions in induced drag and thrust requirements. Ultimately, lower induced drag and thrust translate to higher efficiency and lower fuel costs. The additional structural flexibility of these lightweight materials can, however, lead to aeroelastic interactions that can degrade aerodynamic performance at off-design conditions. Recovering off-design performance through the use of active wing shaping control can maximize the benefits of employing lightweight/ highly flexible aerospace materials in wing structures.

In 2010, a conceptual study titled "Elastically Shaped Future Air Vehicle Concept"1 was conducted by NASA in order to investigate multiple active wing shaping control concepts for flexible wings. The study proposed the Variable Camber Continuous Trailing Edge Flap (VCCTEF) control effector as a means to elastically control the wing washout twist and wing bending deflection to change the local angle of attack to reduce drag. ${ }^{1-3}$ Under the Fixed Wing project Active Aeroelastic Shape Control (AASC) element, NASA and Boeing conducted a joint study to investigate the application and potential of the VCCTEF system. ${ }^{4,5}$ As shown in Fig.1, The VCCTEF is composed of multiple spanwise segments connected by flexible elastomer material. The elastomer material is incorporated as a means to prevent vorticity generation between segments that occurs if a geometric discontinuity exists as in traditional flap

\footnotetext{
*Research Engineer, daniel.chaparroespinoza@nasa.gov

${ }^{\dagger}$ Graduate Student (Ph.D.), Department of Aeronautics \& Astronautics, fujiwar2@uw.edu

${ }^{\ddagger}$ Research Engineer, eric.b.ting@nasa.gov

${ }^{\S}$ Research Scientist, nhan.t.nguyen@nasa.gov, AIAA Associate Fellow
} 
systems. For this study, each spanwise section is made up of three chordwise segments, as shown in Fig.2, that can finely tune the camber of that section at any point within the flight envelope.

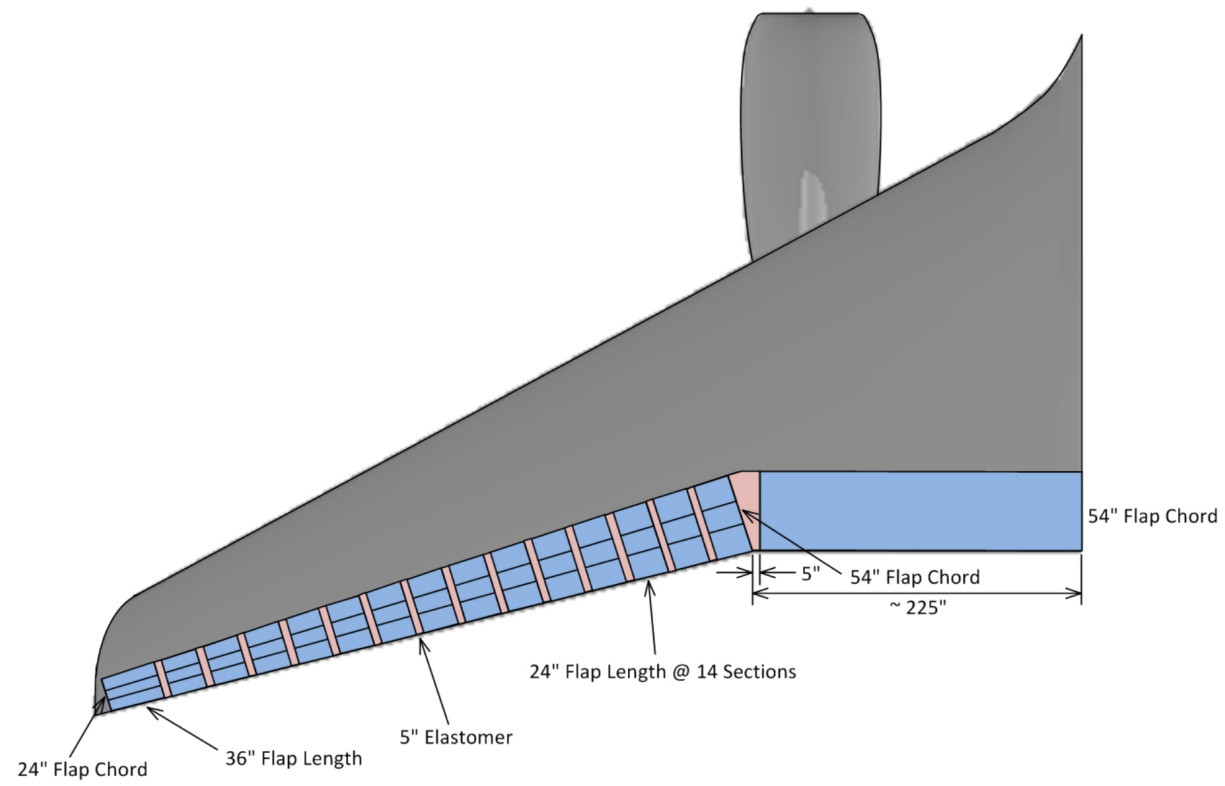

Figure 1. Configuration VCCTEF on a Notional Transport Wing

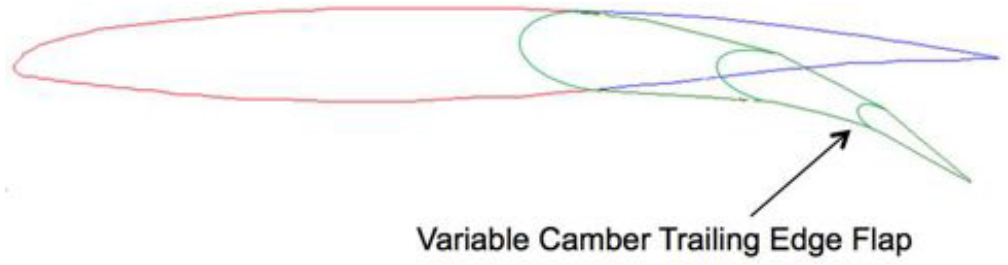

Figure 2. VCCTEF Section with 3 Camber Segments (Green) Compared to a Traditional Flap System (Blue)

The aircraft used in this study is based on the NASA Generic Transport Model (GTM), which is a research model that originated from NASA Langley Research Center. The model was selected because subsonic wind tunnel data was available to validate computational models. The GTM is a 5.5\% dynamically-scaled model of a notional singleaisle, mid-size, 200-passenger aircraft with a similar outer mold line as the Boeing 757. ${ }^{1}$ The design lift coefficient is $C_{L}=0.51$ at the design mid-cruise condition of Mach $=0.797$ at $36,000 \mathrm{ft}$. The GTM equipped with the VCCTEF is shown in Fig.3. 


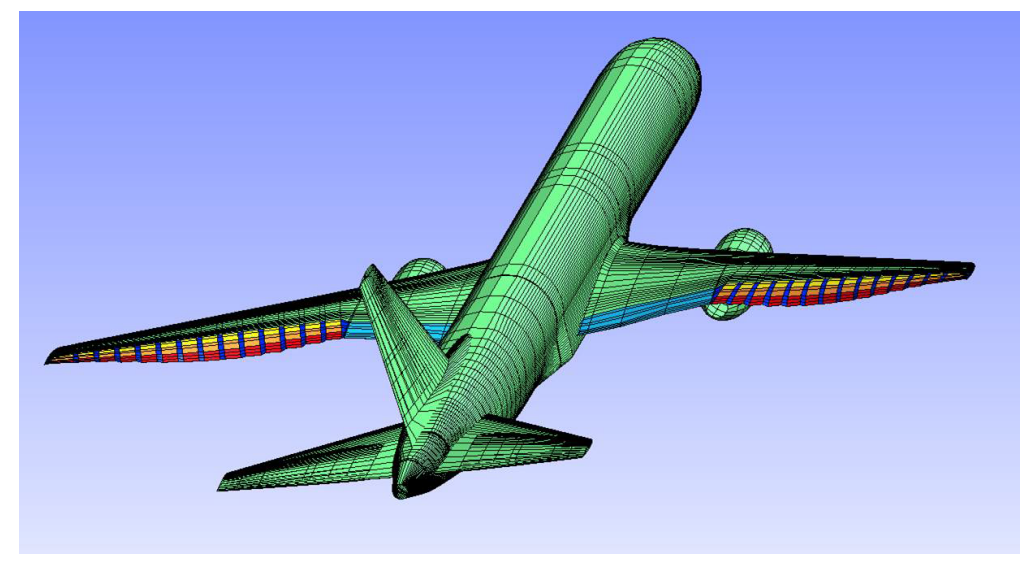

Figure 3. Illustration of GTM Aircraft Equipped with the VCCTEF

Previous studies of the VCCTEF have examined the drag reduction benefits of the VCCTEF by estimating skin friction drag and predicting induced drag through the use of vortex lattice. ${ }^{1,2}$ Since VLM does not account for shock effects, wave drag was not optimized. Since the GTM cruise speed is Mach $=.797$, it is necessary to account for wave drag and transonic effects on lift and induced drag. In a follow up study by Lebofsky, ${ }^{11}$ an Euler solver was leveraged to create two-dimensional lift and drag databases from which to sample and correct the vortex lattice spanwise lift and drag distributions. The study also refined the estimate for skin friction drag by using a flat plate analytical approximation. The framework was efficient and accounted for transonic effects on lift and drag. A limitation of the approach is that it relied on having a database of high-fidelity airfoil data. For example, if one wished to optimize the layout of the VCCTEF, it would be necessary to create an exhaustive database of all the configurations to be evaluated a priori. Creating the database can be time consuming and may yield erroneous predictions for large wing deformations.

This paper builds on the viscous and transonic drag correction proposed by Lebofsky by coupling an open source two-dimensional transonic small-disturbance (TSD) solver, TSFOIL, with vortex lattice to capture wave drag and transonic effects on lift and induced drag. TSD is less computationally expensive than an Euler solver and can thus be incorporated "in the loop" rather than through a predefined database. This paper also details how TSD is coupled with the integral boundary layer model (IBL) developed by Fujiwara $\mathrm{et}^{\mathrm{al}^{26}}$ to capture the attenuation of aerodynamic forces due to boundary layer viscosity. The integral boundary layer model provides a means of predicting skin friction drag and boundary layer separation, which can be used to constrain the settings for the VCCTEF during conceptual design.

\section{Static Aeroelasticity Model}

A static aeroelasticity framework is used to couple the aerodynamics model to a finite-element analysis to appropriately deform the aircraft wing for a given set of operating conditions using an iterative tool similar to past studies. ${ }^{9}, 11,20,37$ A geometry deformer is used in order to aeroelastically deform outer mold line geometry for input into the aerodynamic analysis tool. Figure 4 shows a high level representation of the coupling implemented in the MATLAB aeroelasticity framework. The iterative approach initiates with the aerodynamic model passing sectional load data to the finite element analysis. The finite element analysis then uses the aero loads to calculate translational and rotational vectors along the wingspan. The rotational and translational vectors are used by the geometry deformer to update the outer mold line of the wing and passes the updated geometry back to the aerodynamic tool. The process is iterated until the convergence criteria are met. 


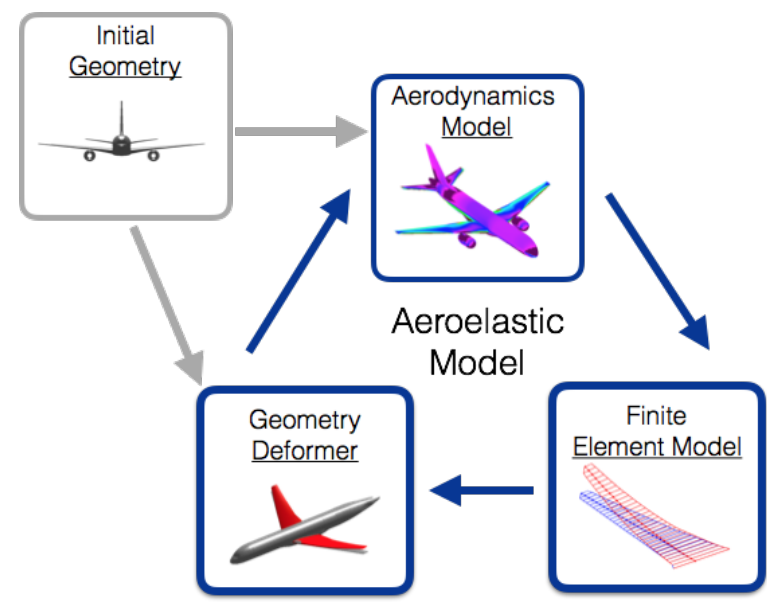

Figure 4. High Level Depiction of the Static Aeroelasticity Modeling Framework

\section{Aerodynamic Model}

The aerodynamic model leverages strip theory to couple the vortex lattice model with 2D TSD+IBL solutions. An overview of the aerodynamic model framework highlighting the major building blocks is shown in Fig. 5. Each major component of the aerodynamic model is described in detail in subsequent sections. The transonic and viscous corrected aero loads are calculated using the following process:

1. The vortex lattice model is executed for the input geometry at the specified input flight condition. Total aircraft aerodynamics are calculated including spanwise distributions of lift coefficient $c_{l}(y)$; drag coefficient $c_{d_{i}}(y)$, quarter-chord moment coefficient $c_{m_{q c}}(y)$; and side force coefficient $c_{y}(y)$.

2. The wing is sliced into streamwise sections and the effective angle of attack and Mach number are calculated for each section.

3. The TSD model is executed for each section. Surface pressure and velocity are calculated for the upper and lower airfoil surfaces.

4. The local pressure and Mach number distributions are used by the IBL model to calculate the boundary layer displacement thickness. The displacement thickness is then added to the airfoil coordinates, effectively thickening the airfoil.

5. The TSD model is executed again for each section using the effective airfoil shape. The resulting surface pressure coefficient is integrated to calculate the sectional lift, drag and pitching moment.

6. The sectional forces are integrated over the span to calculate the total aircraft loads. The sectional aerodynamic loads are passed to the finite element model.

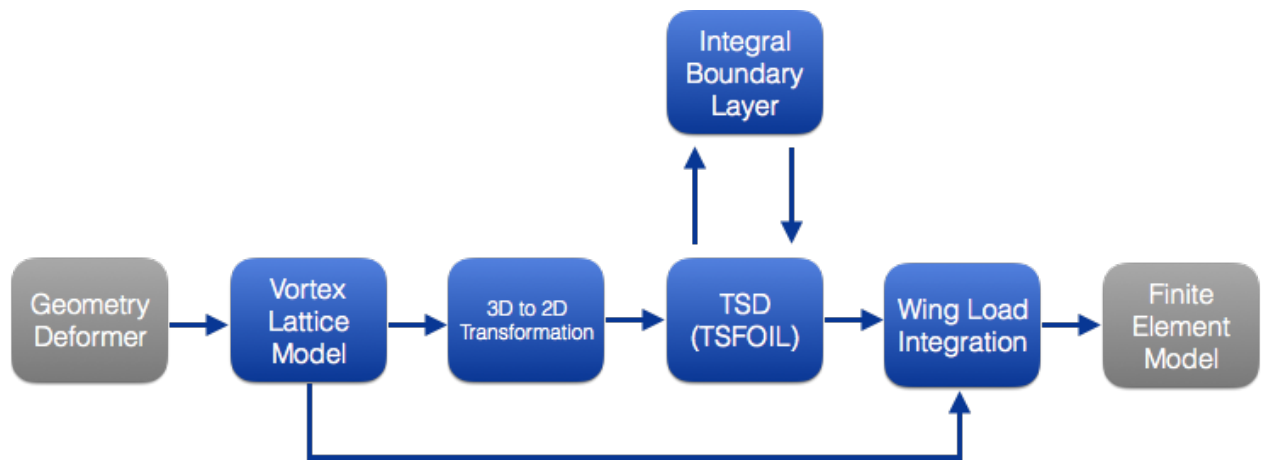

Figure 5. Aerodynamics Model Framework 


\section{A. Vortex Lattice Model}

Vorview is an aircraft aerodynamic modeling tool that uses the vortex lattice method. ${ }^{23}$ The vortex lattice method is an extension of Prandtl's lifting ling theory that is applicable to a broader range of lifting surfaces including swept and low aspect ratio wings. The aircraft geometry is discretized by Vorview into panels along the spanwise and chordwise directions. A horseshoe vortex and a control point are placed at each panel. The strength of each vortex is solved for by requiring that the induced velocity normal to the mean camber of each panel be zero at the control points. Vorview computes the vehicle aerodynamics in both the longitudinal and lateral directions independently as a function of the circulation of each vortex, and these can be combined to produce the overall aerodynamic characteristics of the vehicle at any arbitrary angle of attack and sideslip angle. ${ }^{22}$

Vorview, which is considered a medium-fidelity tool, has been validated by wind tunnel data. ${ }^{21}$ Limitations associated with vortex lattice modeling in general apply to Vorview aerodynamic analysis. Vorview is limited to subcritical or fully-supersonic inviscid flow, consequently, the drag prediction from vorview accounts only for induced drag. Wave drag and viscous drag must be modeled by other methods. Figure 6 shows the the wing-body GTM configuration modeled in Vorview.

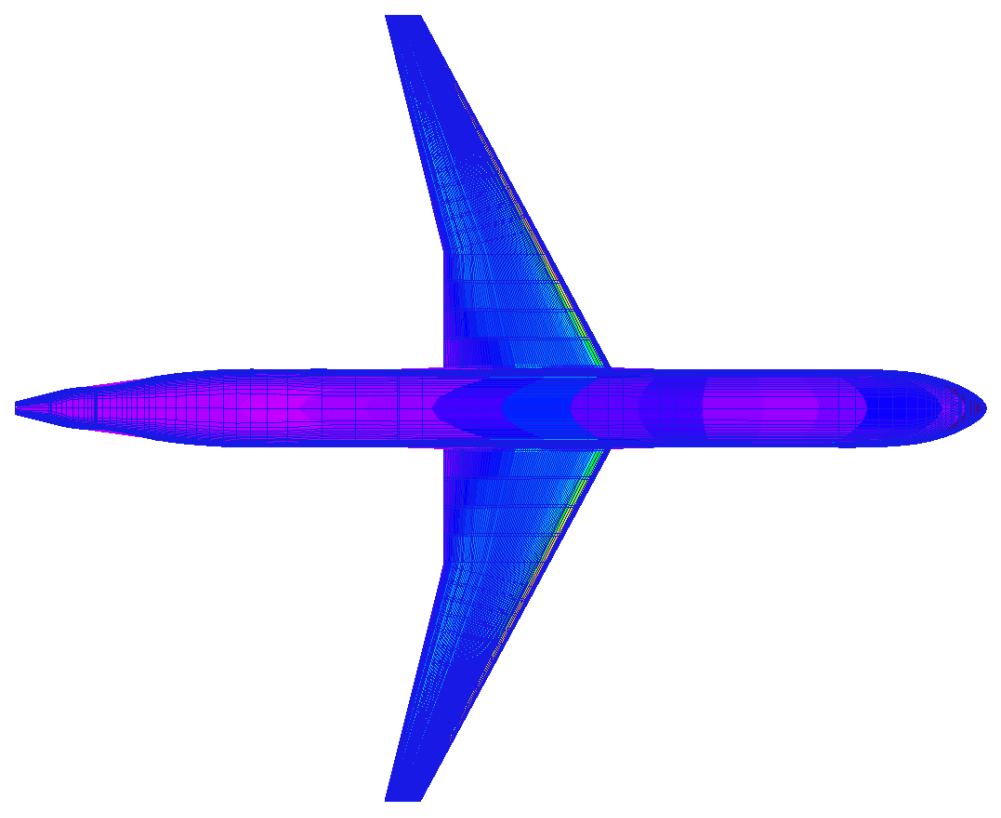

Figure 6. GTM Aircraft Wing-Body Model in Vorview, 90 Spanwise Segments by 22 Chordwise Segments

\section{B. 3D to 2D Transformation}

Streamwise sections along the span of the wing were analyzed using TSD+IBL as shown in Fig. 7. The effective angle of attack and Mach number for the 2D sections must account for 3D effects such as sweep, aspect ratio and finite span. The effective angle of attack for each section was solved for by relating the sectional lift coefficient from VLM, which accounted for 3D effects, with the Prandtl-Glauert corrected theoretical 2D lift coefficient from thin airfoil theory as shown in Eq.1. The Prandtl-Glauert correction is a function of the local 2D Mach number which is the sweep-corrected Mach number with respect to mid chord. The zero lift angle of attack, $\alpha_{0_{2 D}}$, is a function of the airfoil camber and was calculated with the TSD solver.

$$
\begin{gathered}
c_{l_{v l m}}(y)=\frac{2 \pi}{\sqrt{1-M_{\Lambda_{c / 2}}^{2}}}\left(\alpha_{2 D}-\alpha_{o_{2 D}}\right) \\
M_{\Lambda_{c / 2}}=M_{\infty} \cos \Lambda_{c / 2}
\end{gathered}
$$

Where $\alpha_{2 D}$ is the local 2D angle of attack and $y$ is the spanwise distance. 


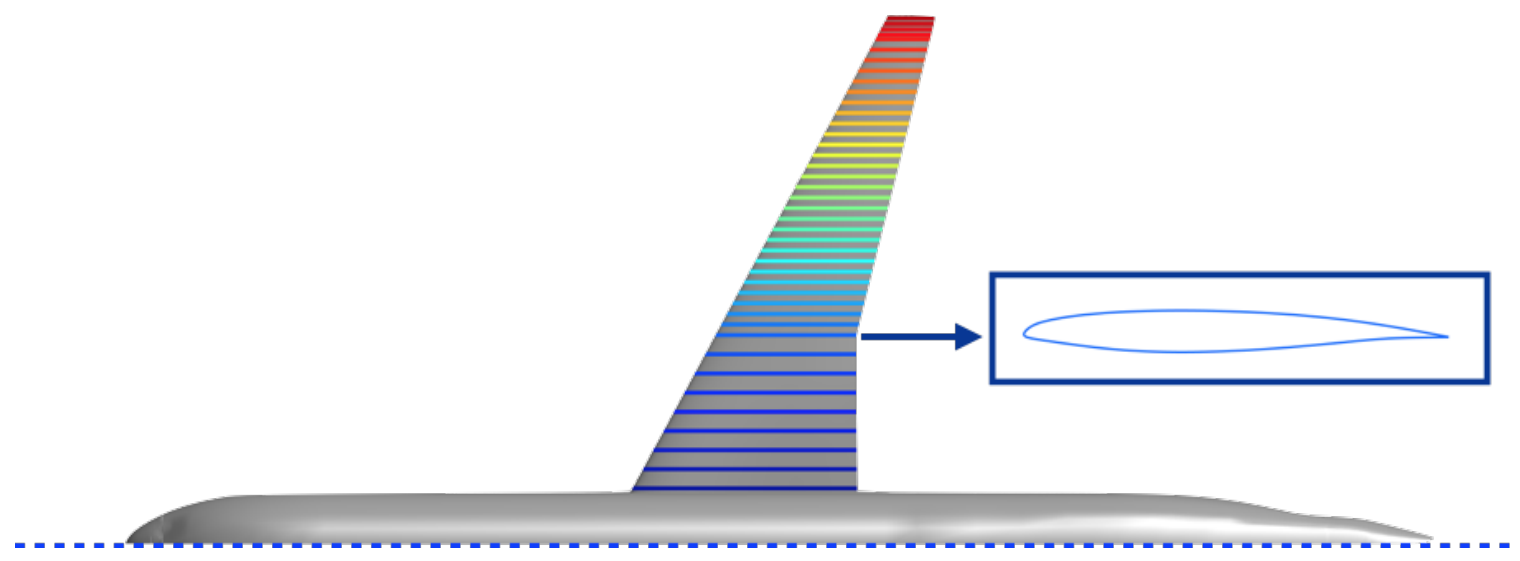

Figure 7. Streamwise Slices of GTM wing for TSD and IBL analysis

\section{Transonic Small-Disturbance Model}

The two-dimensional inviscid aerodynamic analysis for each airfoil section was performed by the TSFOIL solver. TSFOIL is a transonic small-disturbance (TSD) solver for flow past lifting airfoils. ${ }^{25}$ TSFOIL was chosen for its rapid solve time, ease of use, and its open source architecture.

TSFOIL solves for the transonically-scaled perturbation potential, $\phi$, which is defined in terms of the physical perturbation potential, $\Phi$, and similarity parameters as shown in Eq.3

$$
\Phi\left(x^{\prime}, y^{\prime}\right)=c U_{\infty}[x+\Phi(x, y)+\ldots]=c U_{\infty}\left[x+\frac{\delta^{2 / 3}}{M_{\infty}^{n}} \phi(x, \tilde{y})+\ldots\right]
$$

where $\mathrm{c}$ is the airfoil chord, $U_{\infty}$, and $M_{\infty}$, respectively, are the freestream velocity and Mach number. $\delta$ is the airfoil thickness ratio, $(x, y)$ are the physical cartesian coordinates, $\left(x^{\prime}, y^{\prime}\right)$ are normalized by airfoil chord, and $\tilde{y}$ is the transonically-scaled $y$ coordinate. ${ }^{25}$

TSFOIL solves the TSD equation cast in terms of transonic similarity variables. ${ }^{25}$

$$
\frac{\partial}{\partial x}\left[K \frac{\partial \phi}{\partial x}-\frac{\gamma-1}{2}\left(\frac{\partial \phi}{\partial x}\right)^{2}\right]+\frac{\partial^{2} \phi}{\partial \tilde{y}^{2}}=0
$$

where

$$
\begin{gathered}
\tilde{y}=\delta^{1 / 3} M_{\infty}^{m} y \\
K=\frac{1-M_{\infty}^{2}}{\delta^{2 / 3} M_{\infty}^{2 m}}
\end{gathered}
$$

Because of the non-uniqueness of the scaling, various similarity rules are available in TSFOIL and the exponent $m$ depends on the choice of similarity rule used. The options available in TSFOIL are those from Krupp, ${ }^{32}$ Spreiter, ${ }^{33}$ and $\mathrm{Cole}^{34}$ as defined in Table 1 where the exponents are related by Eq. 7. As shown in section IV.A, TSFOIL is in close agreement with Euler solutions when using the Krupp similarity rule.

\begin{tabular}{|c|c|c|c|}
\hline & $\mathrm{k}$ & $\mathrm{n}$ & $\mathrm{m}$ \\
\hline \hline Cole & 0 & 0 & 0 \\
\hline Spreiter & 2 & $2 / 3$ & $2 / 3$ \\
\hline Krupp & $7 / 4$ & $3 / 4$ & $1 / 2$ \\
\hline
\end{tabular}

Table 1. Exponents for Transonic Similarity Parameters

$$
k=2 m+n
$$

$$
6 \text { of } 15
$$


The solver has the option to choose from an analytical mesh, several built in meshes or a custom mesh definition. In an effort to make the framework general, the study employed the analytical mesh within TSFOIL which proved to be robust and provided adequate results. With the analytical mesh, TSFOIL empoloyed a mesh refinement sequence to enhance the rate of convergence. The solution converged on a coarse mesh, continued to a medium mesh before finally reaching a converged solution on the finest mesh. The pseudo-time relaxation factor used in TSFOIL to enhance convergence was set to 0.9 to improve robustness in the framework. Free-air boundary conditions were used. The nonconservative difference scheme across the shock was used to ensure unique solutions from the TSD equation. ${ }^{35}$

It should be noted that TSFOIL is limited to $0.5<\mathrm{M}_{\infty}<2$, not including $\mathrm{M}_{\infty}=1$. However, the shock jumps, as calculated by the program using isentropic small-disturbance theory, will be in error with the true Rankine-Hugoniot value if the normal Mach number just upstream of the shock wave is greater than $1.3 .{ }^{25} M_{n}=1.3$ is usually the accepted upper bound for validity of the isentropic assumption. Because TSFOIL assumes small velocity perturbations, the validity of the solution is suspect for angles of attack greater than nine degrees or highly-cambered airfoils.

TSFOIL writes out surface pressure, $c_{p}$, and Mach number, $M$, for the upper and lower surfaces at each mesh point. $c_{p}$ and $M$ were calculated as a function of the transonically-scaled velocity potential and similarity parameters as shown in Eq.8-9.

$$
\begin{gathered}
c_{p}=\delta^{2 / 3} M_{\infty}^{-n}\left(-2 \frac{\partial \phi}{\partial x}\right) \\
M=\left\{1-\left[K-(\gamma+1) \frac{\partial \phi}{\partial x}\right] \delta^{2 / 3} M_{\infty}^{2 m}\right\}^{1 / 2}
\end{gathered}
$$

$c_{p}$ is then integrated to calculate the aerodynamic coefficients of interest.

$$
\begin{gathered}
c_{n}=\frac{1}{c} \int_{0}^{c}\left(c_{p_{l}}-c_{p_{u}}\right) d x \\
c_{a}=\frac{1}{c}\left[\int_{L E}^{T E} c_{p_{u}} d y_{u}-\int_{L E}^{T E} c_{p_{l}} d y_{l}\right] \\
c_{m_{l e}}=\frac{1}{c^{2}}\left[\int_{0}^{c} x\left(c_{p_{l}}-c_{p_{u}}\right) d x+\int_{L E}^{T E} y_{u} c_{p_{u}} d y_{u}-\int_{L E}^{T E} y_{l} c_{p_{l}} d y_{l}\right] \\
c_{l}=c_{n} \cos \alpha-c_{a} \sin \alpha \\
c_{d}=c_{n} \sin \alpha+c_{a} \cos \alpha
\end{gathered}
$$

\section{Integral Boundary Layer Model}

The integral boundary layer model used in this analysis was developed by Fujiwara et al. ${ }^{26}$ The model employed a direct sequential coupling of the TSD equations to the viscous boundary layer model with special treatment near the trailing edge to avoid Goldstein's singularity due to strong adverse pressure gradients. For a given $c_{p}$ distribution across the airfoil surface, the model marches from the leading edge stagnation point to the trailing edge calculating the boundary layer displacement thickness at each node.

In the laminar region, the model uses the compressible formulation of Thwaites' method ${ }^{27}$ to march the solution towards the laminar to turbulent transition point. Michel's method ${ }^{28}$ is used to locate the transition point. After transition, the compressible formulation of Head's method ${ }^{29}$ is used to march to the trailing edge. The displacement thickness over the aft $5 \%$ of the chord is linearly extrapolated to avoid singular solutions near the trailing edge. Flow separation is determined when the Head shape factor exceeds 2.2. The IBL solution is not valid once the flow is separated; therefore, when flow separation is predicted, the displacement thickness is linearly extrapolated beginning 4\% upstream of the separation point. Further detail of the IBL model can be found in Ref. 26.

The model has been shown to agree well with XFOIL for subsonic flow and with RANS for transonic flow. ${ }^{26}$ 


\section{E. Wing Load Integration}

The sectional lift, wave drag, viscous drag, and pitching moment are integrated across the span to calculate the total wing aerodynamic forces. The induced drag is scaled by the change in lift due to the TSD+IBL corrections squared. The assumption is valid for small flap deflections that do not significantly change the lift distribution across the span.

\section{Onera M6 Model Validation}

The Onera M6 wing transonic wind tunnel test is a classic validation case for computational fluid dynamics solvers. The Onera M6 wing is a simple swept wing of constant symmetrical airfoil section. The wing properties are listed in Table.2. Description of the experiment and CFD validations of the Onera M6 test can be found in Ref. 30.

Table 2. Onera M6 Wing Geometry

\begin{tabular}{|c|c|}
\hline Wing Property & Value \\
\hline \hline Span & $1.196 \mathrm{~m}$ \\
\hline Mean Aerodynamic Chord & $.646 \mathrm{~m}$ \\
\hline Aspect Ratio & 3.8 \\
\hline Taper Ratio & .562 \\
\hline Mid Chord Sweep & $22.9 \mathrm{deg}$ \\
\hline
\end{tabular}
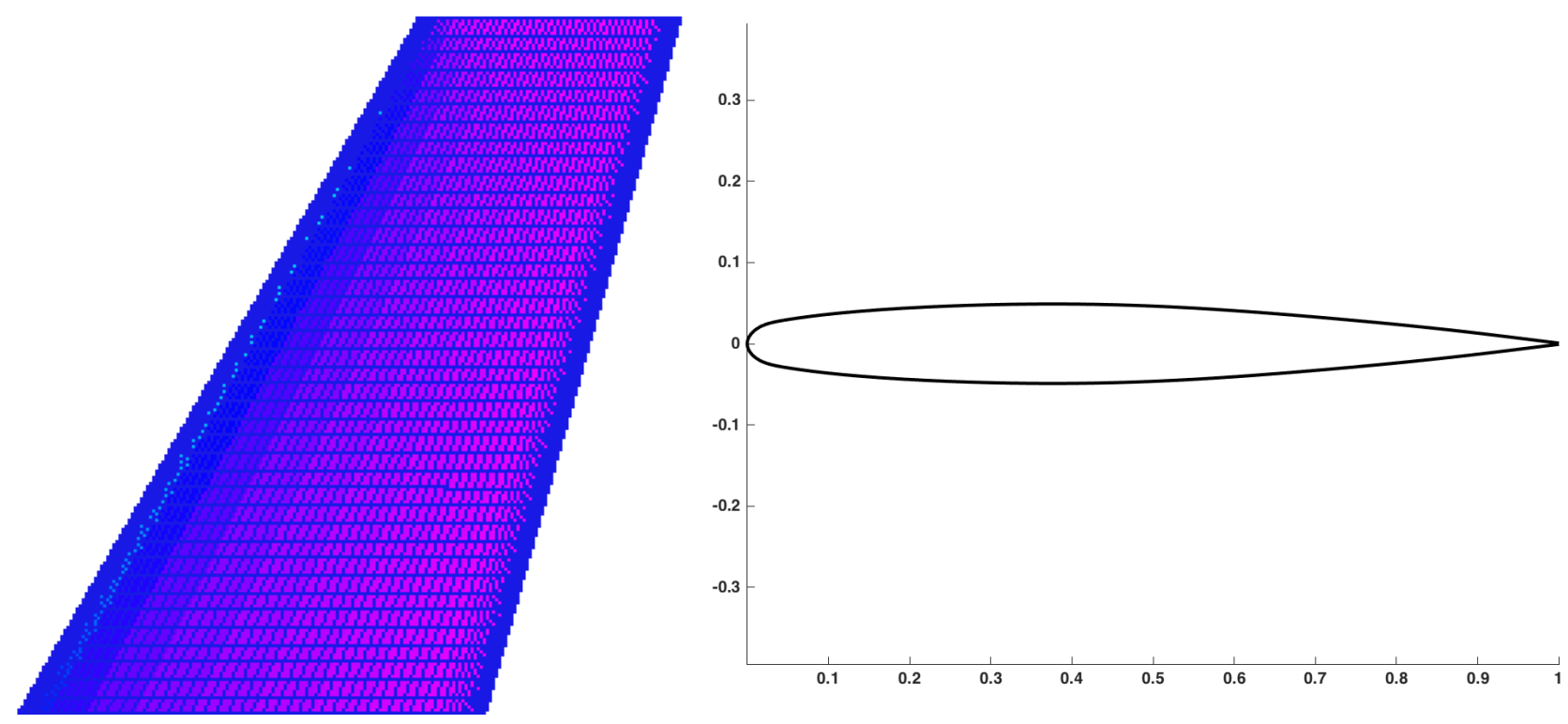

Figure 8. Onera Model in Vorview (Left) and Onera Airfoil Section (Right)

\section{A. Two-Dimensional Comparison of TSD and Euler Solutions}

The Onera M6 airfoil was analyzed in 2D with TSD and compared to two Euler solvers: MSES ${ }^{31}$ and Cart3D. ${ }^{24}$ Figures 9-10 compare lift and drag at $M_{\infty}=0.54$ and $M_{\infty}=0.7$ respectively. The Krupp and Spreiter similarity parameters were examined to determine which rule yielded the best agreement with Euler solutions. As seen in Fig. 9-10 , the TSD -Spreiter similarity rule significantly under predicts lift and drag at both Mach numbers. Consequently, the Krupp similarity rule is used in all further analysis. The TSD- Krupp lift and drag predictions agreed well with the Euler solutions at angles of attack up to four degrees. The TSD-Krupp lift curves deviated from the Euler solutions at high angles of attack where the weak shock assumption was no longer valid. This was evident as TSFOIL produced warnings of $M_{n}$ exceeding the 1.3 limit at $\alpha \geq 6^{\circ}$ and $\alpha \geq 3.5^{\circ}$ for $M_{\infty}=0.54$ and $M_{\infty}=0.7$ respectively. 

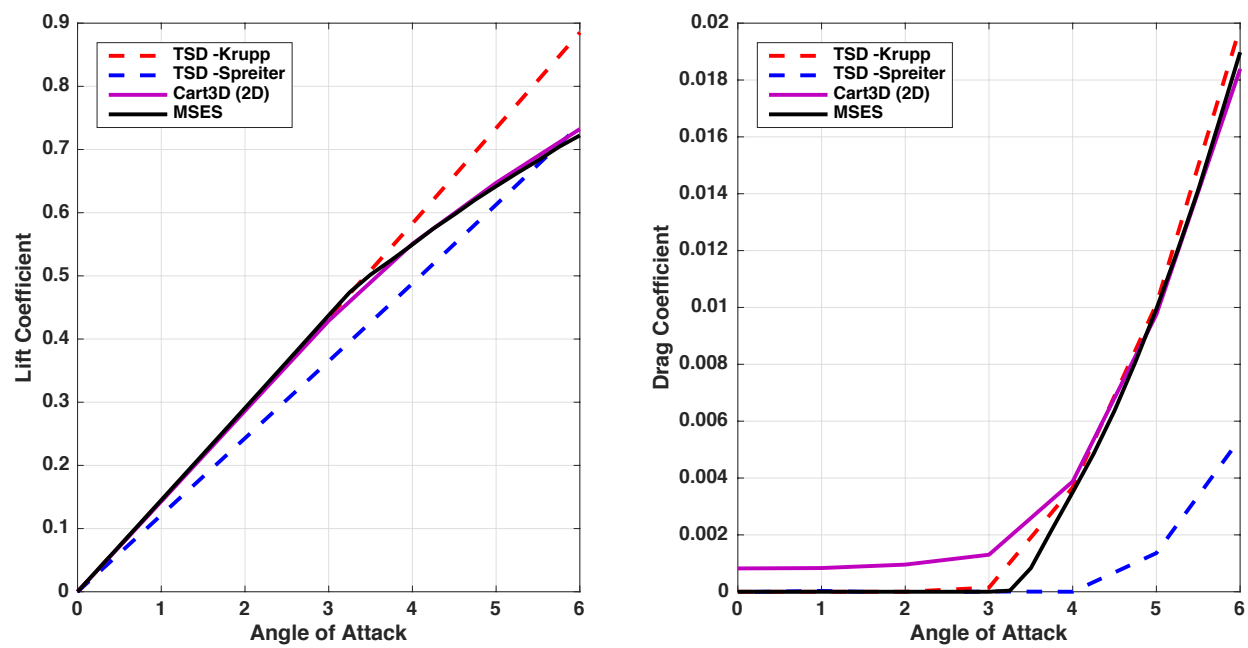

Figure 9. 2D Comparison of Inviscid Lift (Left) and Drag (Right) at $M_{\infty}=0.54$ for the Root Section of the Onera M6 Wing with Simulations using TSD, MSES, and Cart3D (2D)
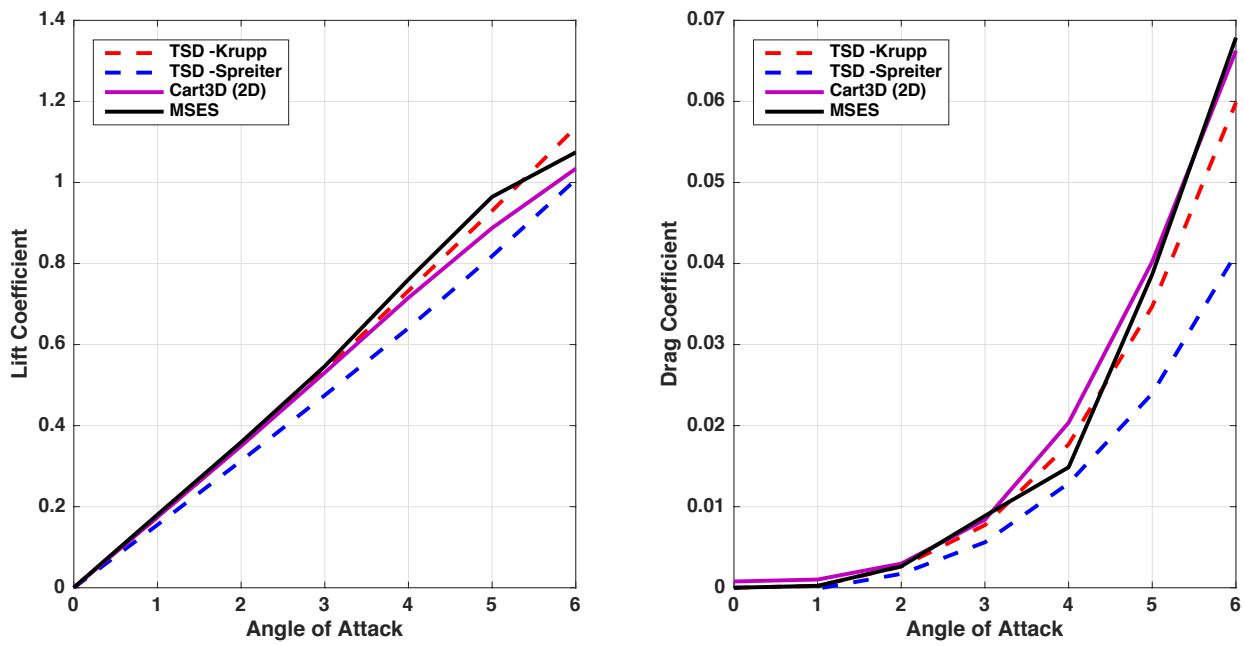

Figure 10. 2D Comparison of Inviscid Lift (Left) and Drag (Right) at $M_{\infty}=0.7$ for the Onera M6 Wing Section with Simulations using TSD, MSES, and Cart3D (2D)

\section{B. Three-Dimensional Coupled VLM and TSD Comparison with Euler}

The Onera M6 wing was modeled in 3D using the VLM+TSD approach and compared to Euler (Cart3D) at $M_{\infty}=0.54$ and $M_{\infty}=0.7$. Whereas the two-dimensional TSD lift did not compare as well with 2D Euler at angles of attack higher than four degrees, the VLM+TSD lift agrees well with 3D Euler solutions at angles of attack up to six degrees. The VLM+TSD drag prediction was in reasonable agreement with Euler. The drag error compared to Cart3D at $\alpha=6 \mathrm{deg}$ was $12.5 \%$ and $13 \%$ at $M_{\infty}=0.54$ and $M_{\infty}=0.7$ respectively. The benefit of the coupled approach was that the TSD model accurately predicted the increase in lift slope due to transonic flow and predicted wave drag which is not otherwise accounted for by VLM alone. 

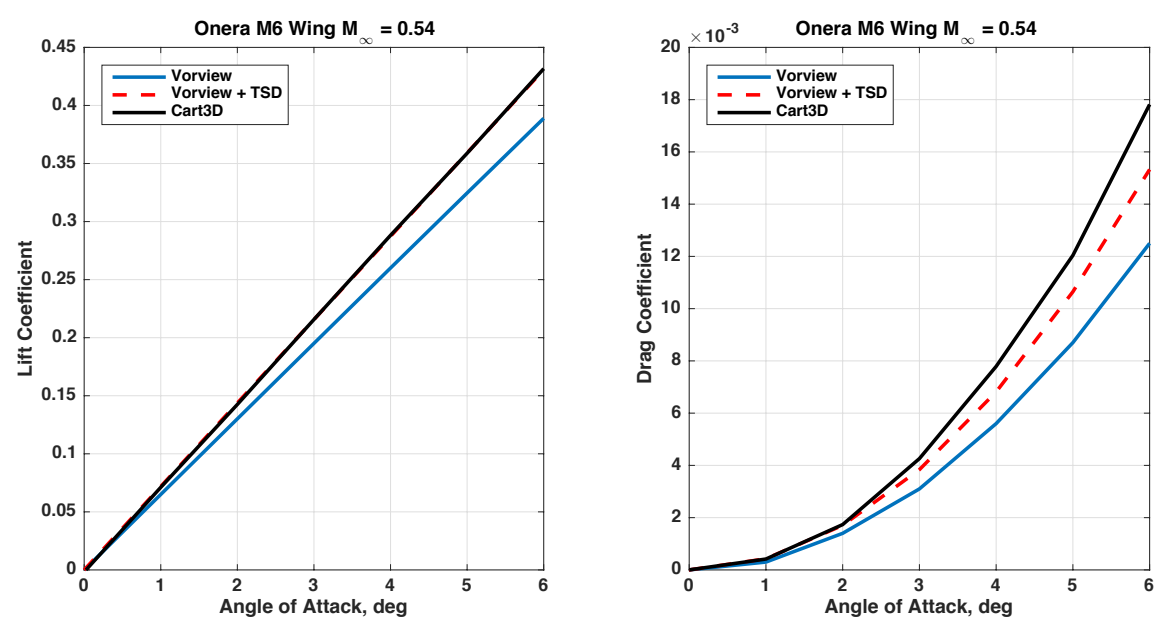

Figure 11. Comparison of Inviscid Lift (Left) and Drag (Right) at $M_{\infty}=0.54$ for the Onera M6 Wing using Vorview+TSD and Cart3D
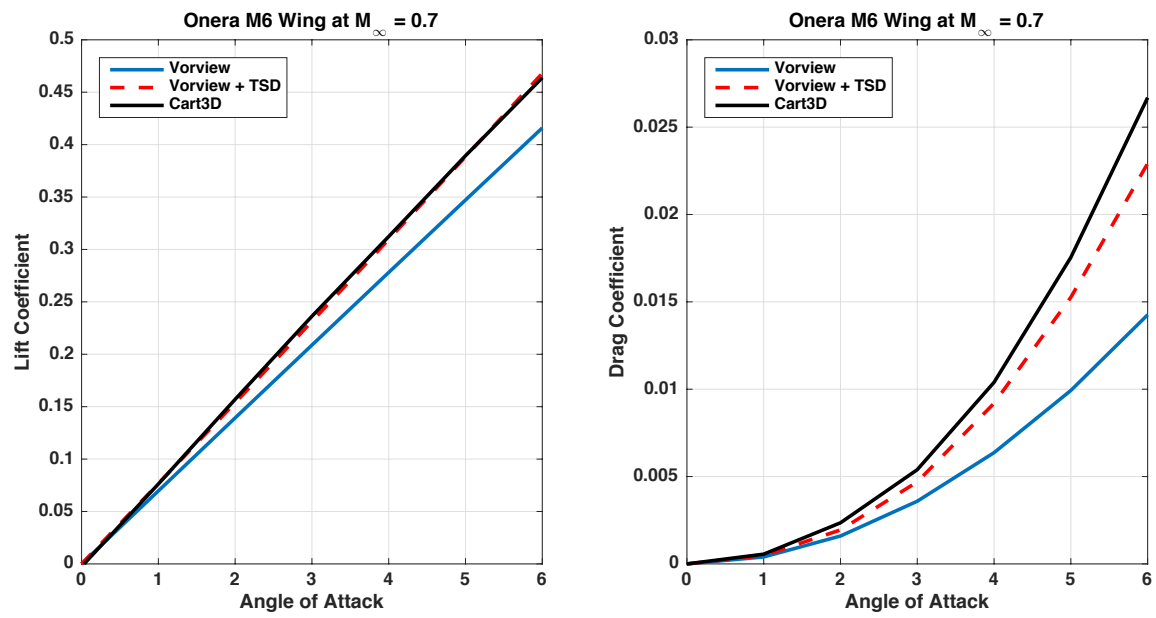

Figure 12. Comparison of Inviscid Lift (Left) and Drag (Right) at $M_{\infty}=0.7$ for the Onera M6 Wing using Vorview+TSD and Cart3D

\section{Results}

\section{A. Rigid Clean Wing GTM Results}

The GTM with a clean wing and no aeroelastic deformation was modeled with the VLM+TSD+IBL framework. Figure 13 shows the predicted lift curve and drag polar. As expected, capturing thickness and transonic effects resulted in a steeper VLM + TSD lift curve when compared to VLM. Conversely, coupling the IBL model reduced the lift slope; thus, the VLM+TSD+IBL lift curve approached the VLM curve. For the clean rigid wing, the result suggests that the viscous effects on lift approximately offset thickness and transonic effects.

The strength of the VLM+TSD+IBL framework is highlighted in the drag polar comparison shown in Fig. 13. The VLM polar only captures the induced drag of the aircraft, while coupling with TSD also captures wave drag resulting in a steeper drag polar. The IBL drag polar shows a nearly constant offset from the TSD drag polar. The constant offset is due to the friction drag predicted by the IBL model, which is approximately insensitive to angle of attack if the flow does not separate. The constant offset suggests that the shock/boundary layer interactions are mild within the analyzed operating conditions. 

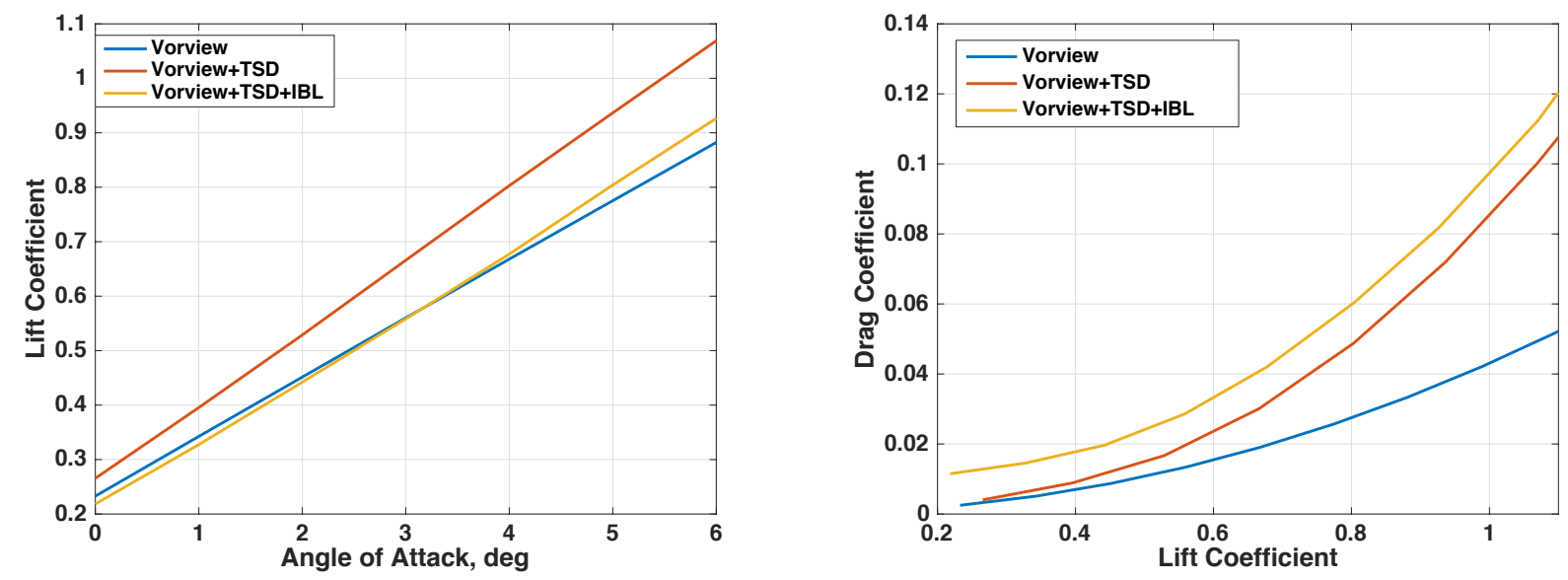

Figure 13. Lift Curve (Left) and Drag Polar (Right) for the GTM using VLM, VLM+TSD and VLM+TSD+IBL Modeling Schemes

\section{B. Static Aeroelastic GTM with VCCTEF Results}

The GTM with the VCCTEF deployed were modeled with the static aeroelastic framework. The elastic properties used in the model are for the half-stiffness GTM wing employed previous studies. ${ }^{11}$ The wing stiffness was also modified to account for varying fuel levels in the wing tanks that correspond to the beginning, middle and end of cruise. ${ }^{11}$ Results with the VCCTEF deployed were compared with clean wing performance. The cruise flight conditions are described in Table 3 and the shape of the VCCTEF configurations are shown in Fig. 14. ${ }^{36}$

\begin{tabular}{ccccc}
\hline & Mach & $C_{L}$ & Altitude $(f t)$ & \% Fuel Load \\
\hline \hline Mid- Cruise & 0.797 & 0.497 & 36,000 & 50 \\
\hline \hline Begin- Cruise & 0.797 & 0.552 & 36,000 & 80 \\
\hline \hline End- Cruise & 0.797 & 0.442 & 36,000 & 20 \\
\hline
\end{tabular}

Table 3. Flight Conditions Analyzed with the VCCTEF Deployed

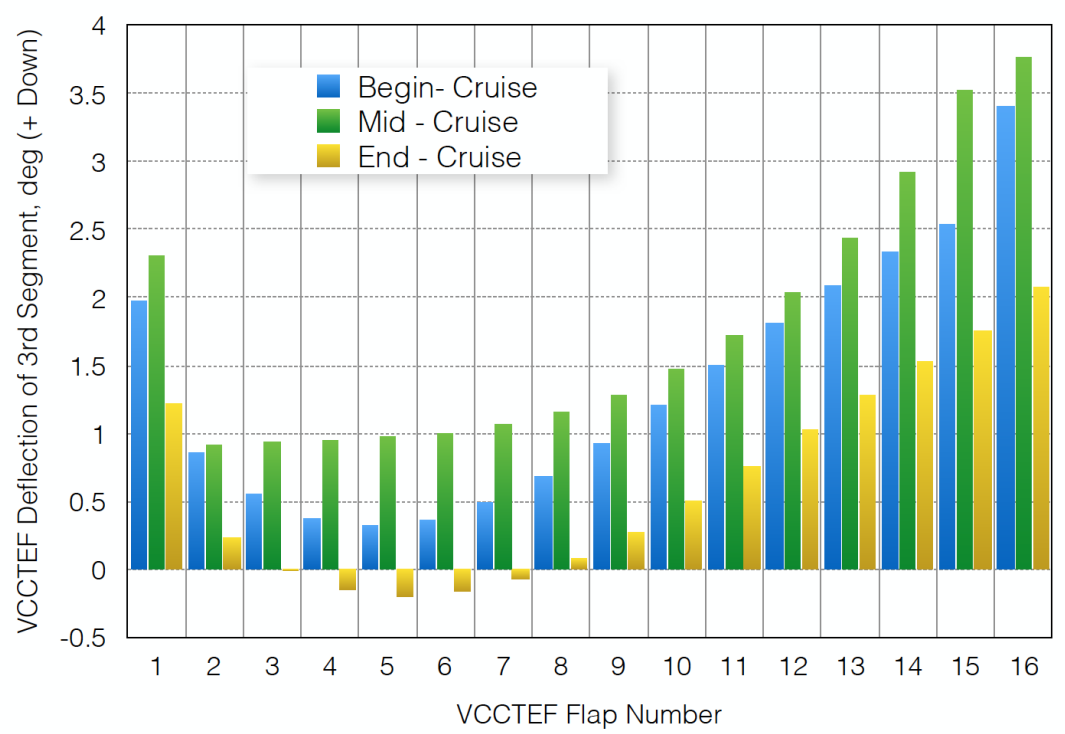

Figure 14. Analyzed VCCTEF Configurations for Begin, Mid, and End Cruise 
As shown in Table 4 through Table 6, deploying the VCCTEF in all of the three analyzed conditions was an effective method of reducing aircraft drag. It is worthwhile noting that the model predicted that the VCCTEF drag reduction was mostly composed of wave drag, which VLM could not quantify without the coupling of TSD. Induced drag was also reduced though it was a relatively small reduction compared to wave drag. A slight friction drag penalty was predicted when the VCCTEF was deployed at mid-cruise and begin-cruise.

\begin{tabular}{cccc}
\hline & Clean Wing & VCCTEF Deployed & Difference \\
\hline \hline Induced Drag (counts) & 112.2 & 112.1 & -0.1 \\
\hline \hline Wave Drag (counts) & 50.6 & 42.9 & -7.7 \\
\hline \hline Friction Drag (counts) & 82.6 & 83.1 & 0.5 \\
\hline \hline Total Drag (counts) & 245.4 & 238.1 & -7.3 \\
\hline
\end{tabular}

Table 4. Drag Reduction with the VCCTEF Deployed at Mid-Cruise

\begin{tabular}{cccc}
\hline & Clean Wing & VCCTEF Deployed & Difference \\
\hline \hline Induced Drag (counts) & 138.5 & 137.4 & -1.1 \\
\hline \hline Wave Drag (counts) & 72.5 & 55.5 & -17.0 \\
\hline \hline Friction Drag (counts) & 82.2 & 82.7 & 0.5 \\
\hline \hline Total Drag (counts) & 293.2 & 275.6 & -17.6 \\
\hline
\end{tabular}

Table 5. Drag Reduction with the VCCTEF Deployed at Begin-Cruise

\begin{tabular}{cccc}
\hline & Clean Wing & VCCTEF Deployed & Difference \\
\hline \hline Induced Drag (counts) & 89.9 & 90.1 & 0.2 \\
\hline \hline Wave Drag (counts) & 36.8 & 33.5 & -3.3 \\
\hline \hline Friction Drag (counts) & 83.4 & 84.4 & 1.0 \\
\hline \hline Total Drag (counts) & 210.1 & 208 & -2.1 \\
\hline
\end{tabular}

Table 6. Drag Reduction with the VCCTEF Deployed at End-Cruise

The drag reduction was seen to vary with lift with the largest improvement seen at begin-cruise. Pressure distribution over the wing is plotted at begin-cruise in Fig. 15 and the strongest shocks are seen near the root. The wave drag reduction is due to the shock weakening effect caused by the VCCTEF. The effect is evident in Fig.16 where pressure distribution for the clean and VCCTEF wing are compared for a streamwise section near the root. 


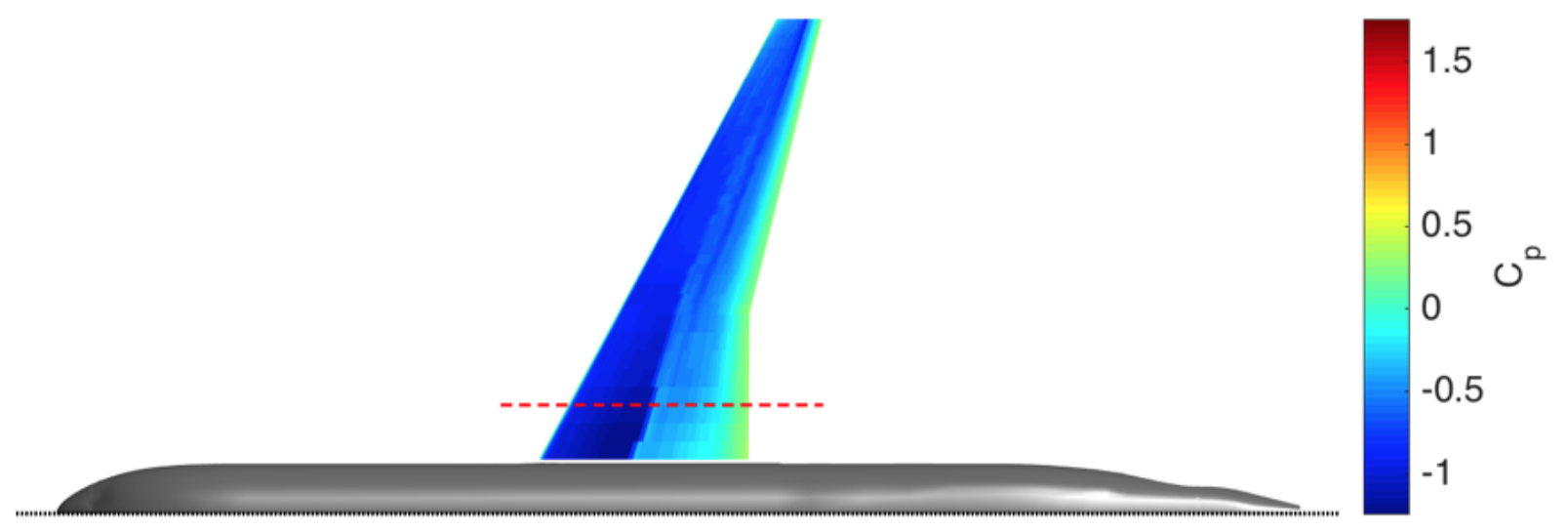

Figure 15. Pressure Distribution Over the Wing for Begin-Cruise Condition with the VCCTEF Deployed

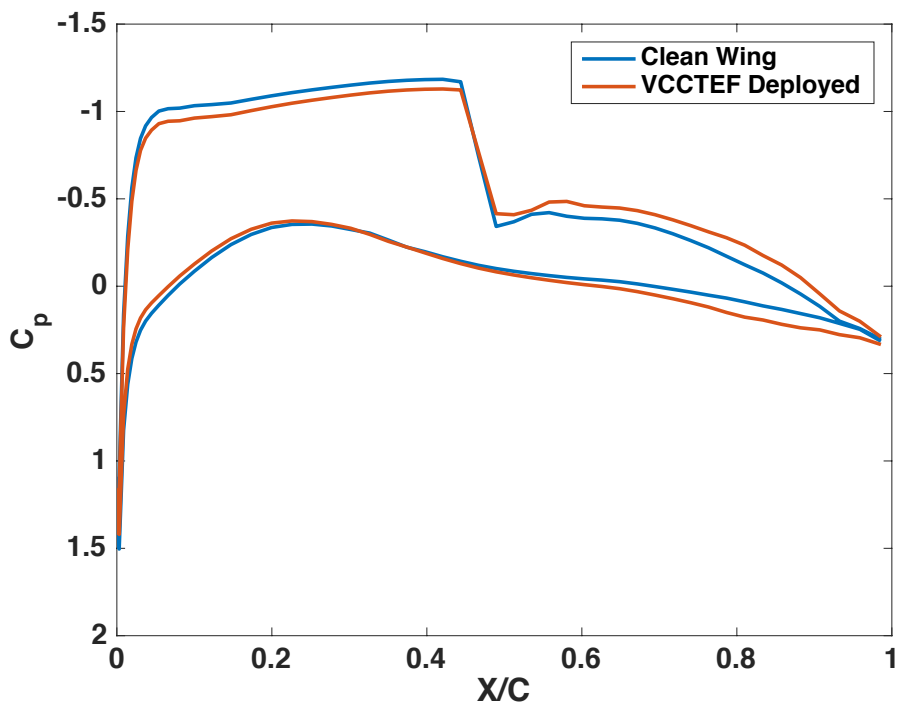

Figure 16. Pressure Distribution Comparison at $\frac{2 y}{b}=0.22$ between the Clean Wing and VCCTEF Wing at Begin-Cruise

\section{Wall-Clock Time Comparison}

The motivation for developing the approach was to develop corrections to VLM that captured more flow physics while maintaining relatively short solve times. Table 7 shows a wall clock comparison between VLM, VLM+TSD and VLM+TSD+IBL, Cart3D.

Note that the calculations were conducted on a Macbook Pro with $16 \mathrm{gb}$ of memory and a $2.5 \mathrm{GHz}$ Intel i7 processor, which has 4 cores/ 8 threads. Unfortunately, VLM does not have the ability to run on multiple cores, but the TSD and Cart3D models were were run on all 4 cores. The VLM+TSD+IBL run time was approximately double the VLM+TSD time because the number of TSFOIL executions approximately doubled when IBL was coupled. Nonetheless, the VLM+TSD+IBL was an order of magnitude faster than Cart3D . The shorter solve time is especially important during conceptual design when there is a need to explore large design spaces with reasonable accuracy.

While it was not practical to run aeroelastic RANS cases for this study on the Macbook Pro, it is worth noting that a single rigid-wing LAVA (RANS) case ran on 320 processors on the Pleiades supercomputer converged in about four hours and the iterative aeroelastic model would require 15-25 executions of the aerodynamic model. 


\begin{tabular}{|c|c|c|}
\hline & Wall-clock time $(\mathrm{sec})$ & Number of Cores \\
\hline \hline VLM & 34 & 1 \\
\hline VLM+TSD & 347 & VLM- 1, TSD -4 \\
\hline VLM+TSD+IBL & 613 & VLM-1, TSD 4, IBL -1 \\
\hline Cart3D (Euler) & 5365 & 4 \\
\hline
\end{tabular}

Table 7. Wall-Clock Comparison of Multiple Aerodynamic Tools per Static Aeroelastic Solution

\section{Conclusions}

A novel approach to correct the vortex lattice method for transonic effects and wing thickness effects using 2D transonic small disturbance solutions was developed and was shown to agree well with the Cart3D Euler solver. The approach was expanded to capture viscous effects using an integral boundary layer model and incorporated into an aeroelastic analysis framework. The fast wall-clock solve times compared to high fidelity RANS and Euler solvers make it attractive for conceptual design studies.

Several VCCTEF configurations were analyzed demonstrating the control effector's ability to reduce drag. Future work will employ the method to explore a wide range of VCCTEF layout configurations with varying number of spanwise and chordwise segments. Ultimately, the approach can efficiently reduce the design space to the point where it is practical to employ high-fidelity analysis tools and fine tune the design.

\section{Acknowledgments}

The authors would like to thank the Advanced Air Transport Technology (AATT) Project under the Advanced Air Vehicles Program (AAVP) of NASA Aeronautics Research Mission Directorate (ARMD) for funding support of this work. The authors also would like to acknowledge the work done by Boeing Research \& Technology (BR\&T) and the University of Washington for their collaboration with NASA on this work under tasks NNL11AD25T and NNL12AD09T entitled "Development of Variable Camber Continuous Trailing Edge Flap System" and "Development of Variable Camber Continuous Trailing Edge Flap System for B757 Configured with a More Flexible Wing".

\section{References}

\footnotetext{
${ }^{1}$ Nguyen, N., "Elastically Shaped Future Air Vehicle Concept," NASA Innovation Fund Award 2010 Report, http://ntrs.nasa.gov/archive/nasa/casi.ntrs.nasa.gov/20110023698_2011024909.pdf, Submitted to NASA Innovative Partnerships Program Office, October 8, 2010.

${ }^{2}$ Nguyen, N., and Urnes, J., "Aeroelastic Modeling of Elastically Shaped Aircraft Concept via Wing Shaping Control for Drag Reduction," AIAA Atmospheric Flight Mechanics Conference, AIAA-2012-4642, August 2012.

${ }^{3}$ Nguyen, N., Trinh, K., Reynolds, K., Kless, J., Aftosmis, M., Urnes, J., and Ippolito C., "Elastically Shaped Wing Optimization and Aircraft Concept for Improved Cruise Efficiency," 51st AIAA Aerospace Sciences Meeting including the New Horizons Forum and Aerospace Exposition, AIAA-2013-0141, January 2013.

${ }^{4}$ Boeing Report No. 2010X0015, "Development of Variable Camber Continuous Trailing Edge Flap System,” October 4, 2012.

${ }^{5}$ Urnes, Sr., J., Nguyen, N., Ippolito, C., Totah, J., Trinh, K., Ting, E., “A Mission-Adaptive Variable Camber Flap Control System to Optimize High Lift and Cruise Lift-to-Drag Ratios of Future N+3 Transport Aircraft," 51st AIAA Aerospace Sciences Meeting including the New Horizons Forum and Aerospace Exposition, AIAA-2013-0214, January 2013.

${ }^{6}$ Nguyen, N., Precup, N., Urnes, J., Nelson, C., Lebofsky, S., Ting, E., Livne, E., "Experimental Investigation of a Flexible Wing with a Variable Camber Continuous Trailing Edge Flap Design," 32nd AIAA Applied Aerodynamics Conference, AIAA 2014-2441, June 2014.

${ }^{7}$ Precup, N., Mor, M., Livne, E., "Design, Construction, and Tests of an Aeroelastic Wind Tunnel Model of a Variable Camber Continuous Trailing Edge Flap (VCCTEF) Concept Wing," 32nd AIAA Applied Aerodynamics Conference, AIAA-2014-2442, June 2014.

${ }^{8}$ Ippolito, C., Nguyen, N., Totah, J., Trinh, K., Ting, E., "Initial Assessment of a Variable-Camber Continuous Trailing-Edge Flap System for Drag-Reduction of Non-Flexible Aircraft in Steady-State Cruise Condition,” AIAA Infotech@ Aerospace (I@ A) Conference, AIAA-2013-5143, August 2013.

${ }^{9}$ Lebofsky, S., Ting, E., Nguyen, N., "Aeroelastic Modeling and Drag Optimization of Aircraft Wing with Variable Camber Continuous Trailing Edge Flap,” 32nd AIAA Applied Aerodynamics Conference, AIAA-2014-2443, June 2014.

${ }^{10}$ Kaul, K., Nguyen, N., "Drag Optimization Study of Variable Camber Continuous Trailing Edge Flap (VCCTEF) Using OVERFLOW,” 32nd AIAA Applied Aerodynamics Conference, AIAA-2014-2444, June 2015.

${ }^{11}$ Lebofsky, S., Ting, E., Nguyen, N., "Multidisciplinary Drag Optimization of Reduced Stiffness Flexible Wing Aircraft With Variable Camber Continuous Trailing Edge Flap," 56th AIAA/ASCE/AHS/ASC Structures, Structural Dynamics, and Materials Conference, AIAA-2015-1408, January 2015.
} 
${ }^{12}$ Ting, E., Dao, T., Nguyen N., "Aerodynamic Load Analysis of a Variable Camber Continuous Trailing Edge Flap System on a Flexible Wing Aircraft," 56th AIAA/ASCE/AHS/ASC Structures, Structural Dynamics, and Materials Conference, AIAA-2015-1839, January 2015.

${ }^{13}$ Nguyen, N., Ting, E., Nguyen, D., Dao, T., Trinh, K., "Coupled Vortex-Lattice Flight Dynamic Model with Aeroelastic Finite-Element Model of Flexible Wing Transport Aircraft with Variable Camber Continuous Trailing Edge Flap for Drag Reduction," AIAA Atmospheric Flight Mechanics (AFM) Conference, AIAA-2013-4746, August 2013.

${ }^{14}$ Tal, E., Nguyen, N., Ting, E., "Comparison of Unsteady Aerodynamics Approximations for Time-Domain Representation of FrequencyIndependent Aeroelastic State-Space Models," 56th AIAA/ASCE/AHS/ASC Structures, Structural Dynamics, and Materials Conference, AIAA2015-1841, January 2015.

${ }^{15}$ Tal, E., Nguyen, N., "Unsteady Aeroservoelastic Modeling of Flexible Wing Generic Transport Aircraft with Variable Camber Continuous Trailing Edge Flap," 33rd AIAA Applied Aerodynamics Conference, AIAA-2015-2722, June 2015.

${ }^{16}$ Swei, S., Nguyen, N., "Aeroelastic Wing Shaping Control Subject to Actuation Constraints," 55th AIAA/ASMe/ASCE/AHS/SC Structures, Structural Dynamics, and Materials Conference, AIAA-2014-1041, January 2014.

${ }^{17}$ Swei, S., Zhu, G., Nguyen, N., "LMI-based Multiobjective Optimization and Control of Flexible Aircraft Using VCCTEF" 56th AIAA/ASCE/AHS/ASC Structures, Structural Dynamics, and Materials Conference, AIAA-2015-1844, January 2015.

${ }^{18}$ Nguyen, N., Tal, E., "A Multi-Objective Flight Control Approach for Performance Adaptive Aeroelastic Wing," 56th AIAA/ASCE/AHS/ASC Structures, Structural Dynamics, and Materials Conference, AIAA-2015-1843, January 2015.

${ }^{19}$ Nguyen, N., Ting, E., Lebofsky, S., "Aeroelastic Analysis of Wind Tunnel Test Data of a Flexible Wing with a Variable Camber Continuous Trailing Edge Flap (VCCTEF)," 56th AIAA/ASCE/AHS/ASC Structures, Structural Dynamics, and Materials Conference, AIAA 2015-1405, January 2015.

${ }^{20}$ Ting, E., Nguyen, N., Lebofksy, S., "Static Aeroelastic Modeling of a Sub-Scale Wind Tunnel Model with Novel Flap Concept," 56th AIAA/ASCE/AHS/ASC Structures, Structural Dynamics, and Materials Conference, AIAA-2015-1407, January 2015.

${ }^{21}$ Nguyen, N., Nelson, A., Pulliam, T., "Damage Adaptive Control System Research Report," NASA Ames Research Center Internal Report, April, 2006.

${ }^{22}$ Ting, E., Nguyen, N., Tringh, K., "Static Aeroelastic and Longitudinal Trim Model of Flexible Wing Aircraft Using Finite-Element VortexLattice Coupled Solution," 55th AIAA SciTech Conference, AIAA-2014-0837, January, 2014.

${ }^{23}$ Miranda, L.R., Elliot, R.D., and Baker, W.M., "A Generalized Vortex Lattice Method for Subsonic and Supersonic Flow Applications," NASA CR-2865, 1977.

${ }^{24}$ Aftosmis, M.J., Berger, M.J., and Melton, J.E., "Robust and Efficient CartesianMesh Generation for Component- Based Geometry," AIAA Journal, Vol. 36, No. 6, 1998, pp. 953-960.

${ }^{25}$ Stahara, S. S., “Operational Manual for Two-Dimensional Transonic Code TSFOIL,” NASA Contractor Report 3064, December, 1978.

${ }^{26}$ Fujiwara, G. E. C., Chaparro, D., and Nguyen, N., "An Integral Boundary Layer Direct Method Applied to 2D Transonic Small-Disturbance Equations," 34th AIAA Applied Aerodynamics Conference, Washington D.C., June 13-17, 2016 (submitted for publication), 2016.

${ }^{27}$ Thwaites, B., "Approximate Calculation of the Laminar Boundary Layer," Aero. Quart., Vol. 1, pp. 245-280, 1949.

${ }^{28}$ Michel, R., "Etude de la Transition sur les Profiles d'Aile; Etablissement d'un Critere de Determination de Point de Transition et Calcul de la Trainee de Profile Incompressible," Tech. rep., ONERA, 1951. Report 1/1578A.

${ }^{29}$ Head, M.R., "Entrainment in the Turbulent Boundary Layer," Aeornautical Research Council Reports and Memoranda Report 3152, September 1958.

${ }^{30}$ Schmitt, V., Charpin, F., "Pressure Distributions on the ONERA-M6-Wing at Transonic Mach Numbers," Experimental Data Base for Computer Program Assessment. Report of the Fluid Dynamics Panel Working Group 04, AGARD AR 138, May 1979.

${ }^{31}$ Drela M., "Newton solution of coupled viscous/inviscid multielement airfoil flows," AIAA Fluid Dynamics, Plasma Dynamics and Lasers Conference, AIAA-1990-1470, June 1990.

${ }^{32}$ Krupp, J. A., "The Numerical Calculation of Plane Steady Transonic Flows Past Thin Lifting Airfoils," Ph.D. Dissertation, University of Washington, 1971

${ }^{33}$ Spreiter, J., "On the Application of Transonic Similarity Rules to Wings of Finite Span," National Advisory Committee for Aeronautics, TR-1153, 1953.

${ }^{34}$ Cole, J. D. and Messiter, A. F., "Expansion Procedures and Similarity Laws for Transonic Flow," Zeitschrift fur angewandte Mathematik und Physik, Vol. 8, No. 1, 1957, pp. 1-25.

${ }^{35}$ Steinhoff, J. and Jameson, A., "Multiple Solutions of the Transonic Potential Flow Equation," 5th AIAA Computational Fluid Dynamics Conference, AIAA 81-1019, June 1981.

${ }^{36}$ Rodriguez, D., Aftomis, M., Nemec, M., Anderson, G., "Optimized Off-Design Performance of Flexible Wings with Continuous TrailingEdge Flaps, " 56th AIAA SciTech Conference, AIAA-2015-1409, January, 2015.

${ }^{37}$ Fujiwara, G. E. C. and Nguyen, N., "Adaptive Aeroelastic Wing Shape Optimization for High-Lift Configurations," AIAA Aviation 2015 Conference, Dallas, TX, AIAA paper 2015-2420, 2015. 